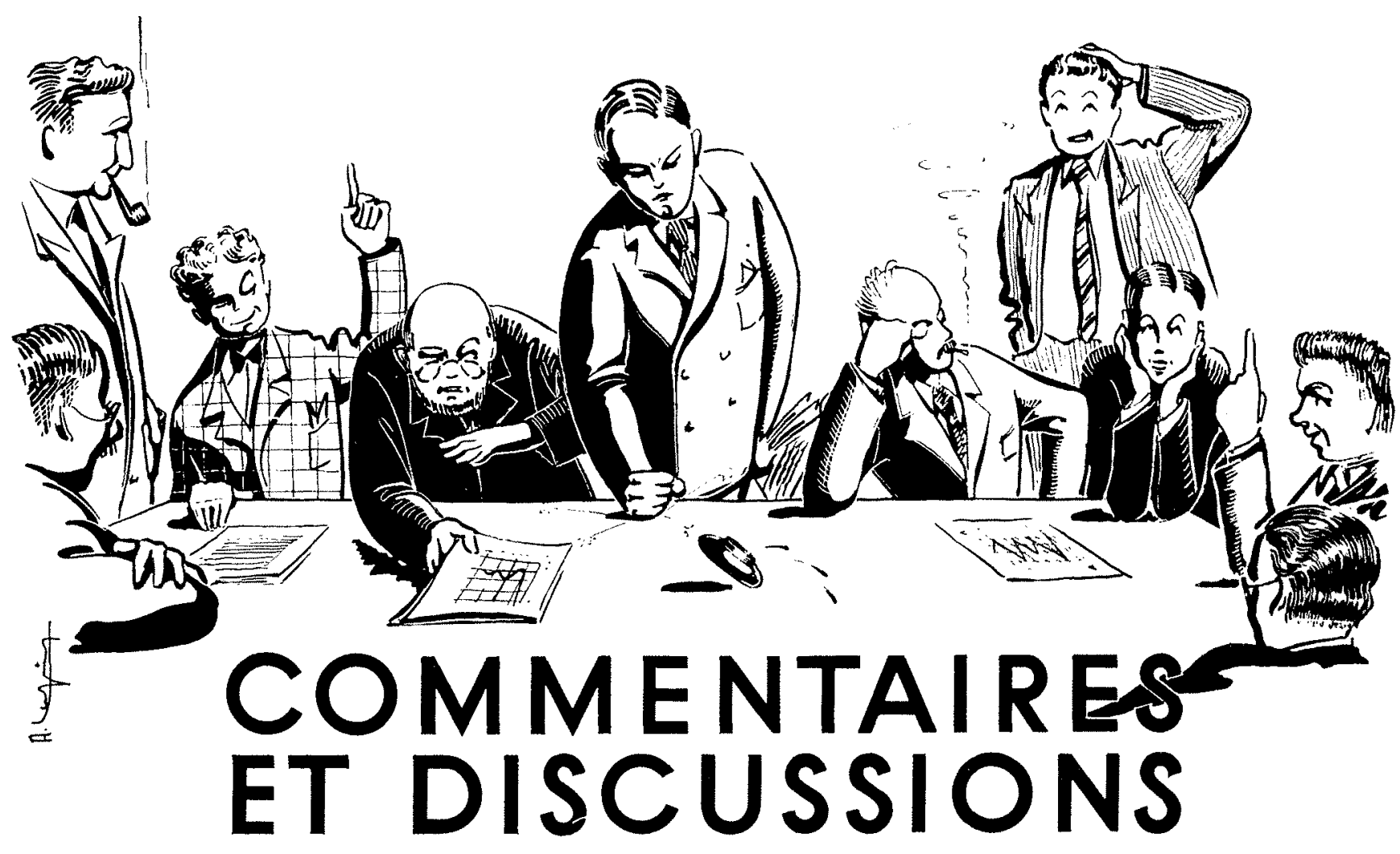

COMMENTS AND DISCUSSIONS

\title{
Écoulement permanent \\ dans les canaux convergents et divergents
}

\author{
Steady flow in contracted \\ and expanded rectangular channels ${ }^{(*)}$
}

Dans l'un des récents numéros de la Homille Blanche a paru un article fort intéressant de MM. ENGilind et MUNCh-PITERSEN concernant les ondes du genre "de Mach» dans les canaux.

Les ondes dans les canaux et, d'une facon générale, les ondes de surface libre, ont un inté-

(*) La Houille Blanche, n* 4, 1953; p. 464. (English $t(x t) ; p .475$ (French text).

(*) Nous avons eu l'occasion de discuter Jonguement de ces problemes avec H. Bresse, ingénieur au Laboratoire Danphinois d'Hydranlique, qui nous a fait part de plusicurs remarques particulierement intéressintes. rèt considerable en hydraulique; rest jourquoi nous croyons devoir vous faire jarl de quelquesunes des remaryues que nous sugsiore (*) l'article en question. Ces remarques peuvent se chasser de la facon suivante:

1. Méthode bémentaire de caleul des angles des ondes de Mach de faible amplitude;

2. Remarques diverses a propos des ondes de Macil:

3. Etude de l'nscuble des perturbations de laibe amplitude dams un eanal fondes de Macu of houle). 


\section{I. -- CALCUL DES ANGLES ET DES ONDES DE MACH PAR UNE METHODE ELEMENTAIRE}

Considérons un bassin très grand de profondeur uniforme, dans lequel coule de l'eau, à la vitesse $u_{m}$, parallèlement à l'axe Ox. En plus, une houle régulière se propage dans ce bassin. Les lignes des crêtes de houle forment un angle $\theta$ avec l'axe $\mathrm{Ox}$. Si la célérité de cette houle par rapport à l'eau $\left({ }^{*}\right)$ est $u_{m} \sin \theta$, les crêtes de la houle semblent immobiles : en effet, elles se déplacent en glissant sur elles-mêmes : figure 1 .

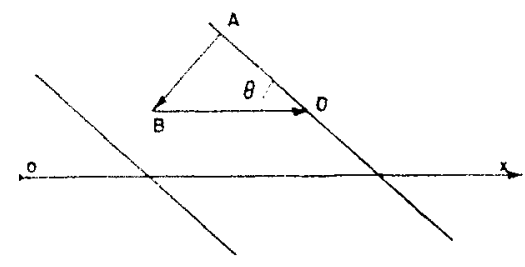

Fig. 1

$A B$ est la vitesse relative de $A ; B D$ la vitesse d'entrainement; $\mathrm{AD}$ est donc la vitesse absolue.

Superposons à cette houle une autre de même célérité (donc de même longueur d'onde), mais dont les lignes de crête sont symétriques des premières par rapport à $O x$ : on obtient une houle gauffrée immobile (fig. 2).

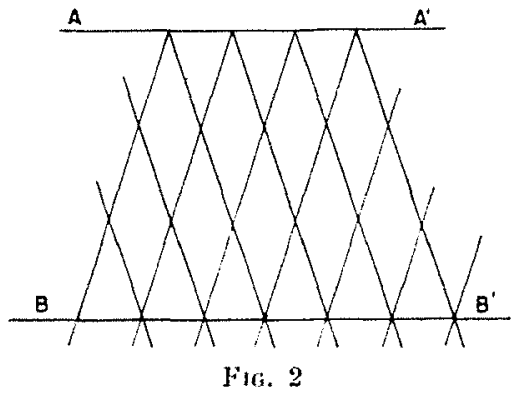

Les lignes $\mathrm{AA}^{\prime}$ et $\mathrm{BB}^{\prime}$ sont des lignes de symétrie de la figure. Sur ces droites, les orbites des houles sont égales et en phase : la vitesse de l'eau y est parallèle à $O x$. On peut donc placer " des tôles » verticales dans le bassin passant par $A A^{\prime}$ et $B^{\prime}$ ' sans perturber l'écoulement (principe de métallisation bien connu en électricite). On constitue ainsi un canal dans lequel il existe des ondes de Mach. La largeur $D$ de ce canal est liée à la longueur d'onde $L$ et à l'angle $\theta$ : fi-

(*) Rappelons que la houle, comme toutes les ondes, se propage à célérité donnée par rapport au milleu dans lequel elles prennent naissance et non par rapport à un systeme d'axes fixes. gure 3. On a $\mathrm{BC}=\mathrm{L}$ : longueur d'onde; $\mathrm{DE}=b / n ; n$ entier, $b$, largeur du canal.

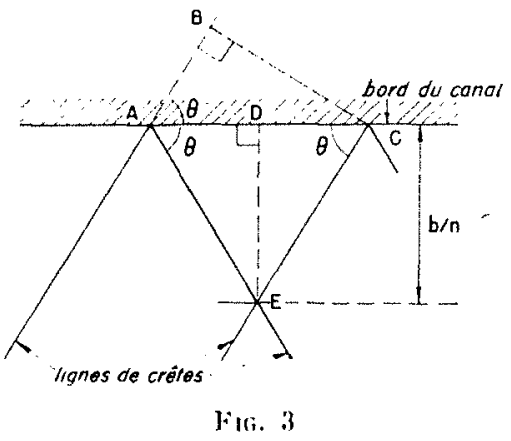

D'où :

$$
\mathrm{AD}=\mathrm{ED} \operatorname{ctg} \theta=\frac{b}{n} \operatorname{ctg} \theta
$$

at :

$$
A D=\frac{A C}{2}=\frac{B C}{2} \frac{1}{\sin \theta}
$$

En égalant les deux valeurs de $A D$, on oblient :

$$
\mathrm{L}=2 \cdot \frac{b}{n} \cos \theta
$$

Celte relation donne avec:

$$
c=u_{m} \sin \theta
$$

et:

$$
c=\sqrt{\frac{g \mathrm{~L}}{2 \pi}} \text { th } \frac{2 \pi y_{m}}{\mathrm{~L}}
$$

(formule bien connue de la célérité de la houle).

$$
\sin \theta=\frac{\sqrt{g y_{m}}}{a_{m}} \sqrt{\frac{\operatorname{th}\left(\frac{n \pi y_{m}}{b} \frac{1}{\cos \theta}\right)}{\frac{n \pi y_{m}}{b}-\frac{1}{\cos \theta}}}
$$

Si on fait $n=2$, on obtient la formule (7) page 467 de l'article cité. $n$ quelconque donne la formule annoncée par les auteurs en haut de la page 468 . Les notations sont les mêmes dans les deux études.

On peut facilement démontrer que, pour chaque valeur de $n>0$, il existe un et un seul angle de Mach. 
On pose à cet effet :

$$
\sin \theta=\mathrm{Y} ; \frac{2 \pi y_{m}}{b} \frac{1}{\cos \theta}=\mathrm{X} .
$$

Les valeurs de $\mathrm{X}$ et $\mathrm{Y}$ sont données par lintersection de :

$$
\mathrm{Y}_{1} \underline{x}=\frac{1}{\mathrm{~F}} \cdot \frac{\mathrm{th} n \mathrm{X}}{n \mathrm{X}}
$$

et :

$$
\mathrm{Y}_{2}{ }^{2}=1-\frac{p^{2}}{\mathrm{X}^{2}}
$$

Ces deux courbes sont symétriques par rapport à l'axe $\mathrm{OY}$. Pour $\mathrm{X}>0$, la premiere décroît toujours, la seconde croit toujours. De plus, $Y_{1}>Y_{2}$ pour $X=0$, et $Y_{1}<Y_{2}$ pour $X=\cdots$.
II n'y a done qu'une racine $Y$. Or, $Y_{1}$ est toujours positif et $Y_{2}$ toujours inférieur à 1 , done. il existe un angle $\theta$ et un seul compris culre 0 et $\pi / 2$ ) qui satisfait l'équation proposée. On peut remarquer que $n=0$ peut donner une onde possible. Dans ce cas, $\theta=\pi / 2$. Mais cette onde. n'existe que si $\mathrm{F} \leqslant 1$; comme on voit aisément :

$$
u_{m}=\sqrt{\frac{g \mathrm{~L}}{2 \pi}} \text { th } \frac{2 \pi y_{m}}{\mathrm{I}} \leqslant \sqrt{g \eta_{m}}
$$

Si $n_{m} \leqslant \sqrt{g y_{m}}$ (c'est-a-dire $\mathrm{F} \leqslant 1$ ), il existe une longueur d'onde $I$. (et une seule) satisfaisant l'équation.

Nous démontrerons au paragraphe III que les ondes ainsi trouvées sont les seules qui puissent exister.

\section{II. - REMARQUES DIVERSES A PROPOS DES ONDES DE MACH}

\section{Le ressidut.}

Dans la théorie classique, le ressaut est une onde de $\mathrm{MACH}$ spéciale correspondant à $\mathrm{F}=1$. Elle est liée à l'onde obtenue pour $n=0$. En réalité, on sait que le ressaut ne peut pas être obtenu avec les équations linéarisées: par contre, on peut calculer de cette facon les ondulations qui peuvent accompagner le ressaut (voir l'article de M. R. Lemorne dans la Houllle Blanche, $\mathrm{n}^{n} 2-1948, \mathrm{p} .183$ ).

\section{2. - Canat de canacténistigues variamis.}

D’après ce qui précède, il semblerait, à première vue, que la direction des ondes de MACH ne dépende, en première approximation, que de la hauteur de l'eau et de la vitesse en chaque point. C'est d'ailleurs ce qu'on admet dans la théorie classique. $\mathrm{Or}$, en étudiant les houles dans les canaux à fond incliné, on s'aperçoit que

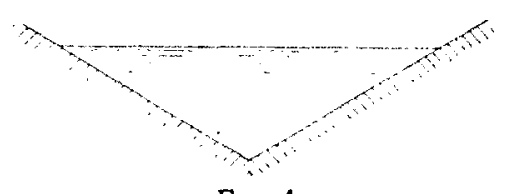

Fig. 4

Conpe d'un canal it ondes de Mach rectilignes

le problème est bien plus compliqué. En effet (voir par exemple dans le livre de $\mathrm{H}$. Lamb : $\mathrm{H}_{\text {/- }}$ dronynamics, édition de 1945 , p. 448 et suivantes): les ondes de Mach déduites de la houle sont parfaitement rectilignes, même dans un canal ì berges inclineses (fig. 4), alors que si leur angle dépendait de la hauteur locale, elles devaient ètre courbes. Néanmoins dans certains cas la théorie classique donne bien des résultats conformes à l'expérience. Une étude approfondie serait nécessaire pour trancher ce litige.

\section{3. - Raideur des ondes de Mach.}

Considerrons un écoulement tel que celui représenté sur la figure 5.

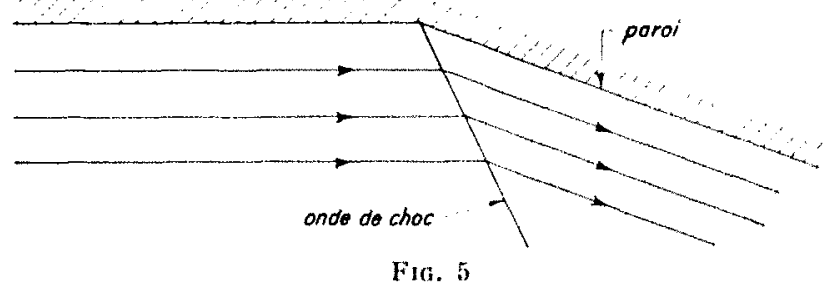

Dans un écoulement gazeux on sait qu'il n'existe qu'un seul angle d'onde de choe, th que cetle onde est effectivement très raide. Par contre, d'après ce qu'on vient de voir, il existe en hy-

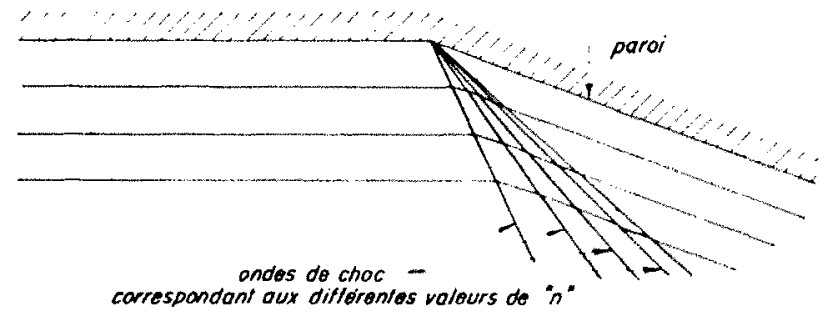

FIG, 6 
draulique un grand nombre d'ondes de choc ayant des angles différents. On doit donc s'attendre à un écoulement du genre de celui représenté sur la figure 6 . Il est trìs probable que l'onde de choc est moins raide et en quelque sorte italée. C'est d'ailleurs ce qu'on observe. La théorie exacte serait très compliquée.

\section{4. -..- SIGNIFICATION ET GHANDEUR REATTIVI DES DIFFÉRENTES ONDES.}

Comme on le verra plus en détail au paragraphe III, les différentes ondes proviennent des perturbations imposées aux deux extrémités du canal. Dans les sections extrèmes, la somme des ondes doit être égale à ces perturbations. Or, pour chaque onde de $\mathrm{MAcH}$, la variation de $u, v$ ou $\Phi$ (composantes de vitesses et potenliel) suivant $\mathrm{O} y$ dépend de l'ordre de l'onde; clle est du genre $\cos (2 \pi n y / b)$. Si la variation de la perturbation est relativement lente suivant O $y$, les harmoniques d'ordre élevé ont des ampliludes tris pelites et seules les ondes d'ordre très bas sont importantes. C'est bien ce que semblent indiquer les essais relatifs aux figures $3^{\mathrm{B}}$ et $8^{\mathrm{A}}$ de l'article cité plus haut. Dans la théorie classique, il existe aussi des ondes d'ordre différent.

[on a :

$\Phi=\stackrel{D}{x=0}_{k=0}^{\infty} \cos \frac{k \pi y}{b} \cos \left(\frac{k \pi}{b} \frac{x}{\sqrt{1 \cdots-F}}+\varphi_{k}\right)$

a comparer au résultat du paragraphe 31 , mais leur angle de Macr est le même; leur distinclion at donc moins d'importance.

\section{5. - RÉgrme torRentiel ét RÉgine Fluvial.}

On verra au paragraphe III que la célérité des ondes obliques dans un canal est plus grande que celle des ondes à crêtes perpendiculaires à laxe; les premières peuvent donc remonter dans un écoulement même torrentiel. L'écoulement torrentiel n'est donc pas un écoulement dans lequel aucune onde ne peut remonter le courant, mais seulement dans lequel aucune onde à crête perpendiculaire à l'axe du canal ne peut remonter le courant. En fait, cette remarque n'a qu'une portée pratique très limitée.

\section{6. - Vagues de pente.}

On sait que la théorie des ondes de faible amplitude en canal de faille profondeur rend compte de l'instabilité des écoulements pour lesquels $U>k c$ ( $k$ dépend de la variation du froltement en fonction de la hauteur). Il apparaît, dans ce cas, des ondes qu'on appelle des « vasues de pente ». Ces ondes ne sont d'ailleurs possibles qu'en canal rectiligne, et bien calibré. Dans le cas contraire, elles sont rapidement détruites par des ondes « de travers 》 dans le canal. Ces ondes ayant une célérité plus grande ruc lins ondes ordinaires ne satisfont pas à $\mathrm{U}>$ kc.

\section{III. - ETUDE DE L'ENSEMBLE DES PERTURBATIONS DE FAIBLE AMPLITUDE POUVANT EXISTER DANS UN CANAL PRISMATIQUE}

\author{
1. - CAS dES ONDES DE MAGH QVAND La vitesSE est \\ PARTOUT CONSTANTE DANS LE CANAL.
}

Considérons le canal trapézö̉dal de la figure 7 . Si le fluide est parfait ef l'écoulement irrotation-

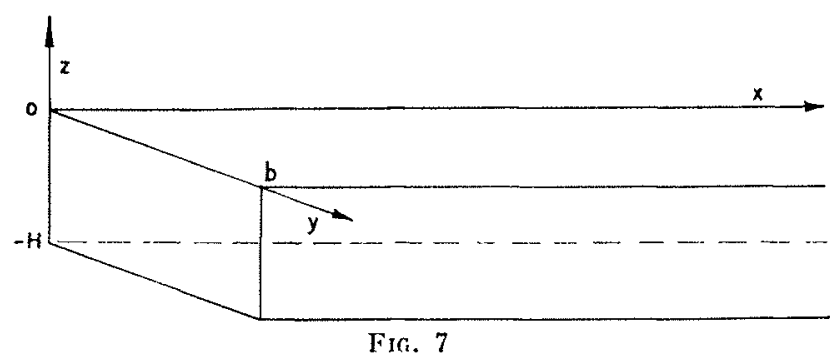

nel, les perturbations de faible amplitude satisfont aux éfuations suirantes (voir par exemple IAMB, loc. cil., p. 363 et suivantes).

$$
\begin{gathered}
\overrightarrow{\mathrm{V}}=\overrightarrow{\mathrm{grad}} \Phi \\
\Delta_{3, \Phi=0}\left(\Delta_{3}=\frac{\partial 2}{\partial x^{2}}+\frac{\partial^{2}}{\partial y^{2}}+\frac{\partial^{2}}{\partial z^{2}}\right)
\end{gathered}
$$

$\frac{\partial \Phi}{\partial n}=0$ sur les parois et an fond. En plus, il existe une condition a la surface libre qu'on déduit facilement de la relation de PoIssox :

$$
g \frac{\partial \Phi}{\partial z}+\frac{\partial^{2} \Phi}{\partial t^{2}}=0
$$

Celle-ci est valable quand l'eau est au repos et les perturbations en mowement. Si on fait un changement d'axes $x^{\prime}=x-\mathrm{V} t$, on obtient facile- 
ment la condition pour les perturbations fixes dans un écoulemenl de vitesse V:

$$
g \frac{\partial \mathrm{W}}{\partial z}=\mathrm{V}^{2} \frac{\partial^{2} \mathrm{~W}}{\partial x^{2}}
$$

On peut facilement résoudre le probleme par la méthode des produits de fonctions orthogonales.

On pose :

$$
\Phi(x ; y ; z)=\Lambda(y) \cdot \mathrm{B}(x ; z)
$$

D'où :

$$
\frac{\mathrm{A}^{\prime \prime}}{\mathrm{A}}=-\frac{\Delta_{2} \mathrm{~B}}{\mathrm{~B}}=\lambda\left(\Delta_{2}=\frac{\partial^{2}}{\partial y^{2}}+\frac{\partial^{2}}{\partial z^{2}}\right)
$$

On sait (voir par exemple le traité classique de Hilbert : Probleme der Mathematischen Physik, tome I, page 310 et suivantes), qu'il suffit dans le cas présent de considérer 2. réel.

On obtient alors:

$\lambda>0 \quad \mathrm{~A}=e^{\sqrt{\lambda} y} \quad$ ou $\quad e^{-\sqrt{\lambda} y}$

$i<0 \quad A=\cos \sqrt{-i} y$ ou $\sin \sqrt{-i} y$

$\lambda=0 \quad \mathrm{~A}=y, \quad$ ou 1

La condition $\frac{\partial \Phi}{\partial n}=0$ sur les parois exige $\lambda \leqslant 0$.

Posons :

$$
\lambda=-u .^{2}
$$

l'où :

$$
A=\cos y \cdot y \quad \text { ef } \quad y \cdot b=k \pi \quad(k: \text { entier })
$$

Done :

$$
\begin{gathered}
\mathrm{A}=\cos \frac{k \pi y}{b} \\
\mathbf{D}=\sum_{k=0}^{\infty} \mathrm{C}_{k} \cos \frac{k \pi y}{b} \mathrm{~B}(x ; z)
\end{gathered}
$$

avec :

$$
\Delta_{2} B=\frac{l^{2} \pi^{2}}{b^{2}} \quad B
$$

On pose the même :

$$
\mathrm{B}=\mathrm{D}(x) \cdot \mathrm{E}(z)
$$

d'oil :

$$
\frac{\mathrm{E}^{\prime \prime}}{\mathrm{E}}=-\left(\frac{\mathrm{D}^{\prime \prime}}{\mathrm{D}}-\frac{k^{2} \pi^{\pi_{2}}}{b^{2}}\right)=
$$

$y>0 \quad \mathrm{~F}=e^{\sqrt{r} r}$ oll $e^{-\sqrt{r} r}$

$v>0 \quad \mathbf{A}=\cos \sqrt{\ldots y} z$ oul $\sin \sqrt{-v z}$

$y<0 \quad \mathrm{E}=z$ ou 1
La condition de fond $\frac{\partial w}{\partial n}=0$ exige :

$v<0 \quad \mathrm{li}=-\operatorname{ch} \sqrt{\mathrm{v}}(z+\mathrm{H})$

$v=0 \quad \mathrm{E}=\cos \sqrt{v}(z+H)$

$v=0 \quad \mathrm{E}=1$

La condition de surface libre devient :

$$
g \frac{\partial \mathrm{E}}{\partial z} \mathrm{D}+\mathrm{V} * \mathrm{E} \mathrm{D}^{\prime \prime}=0
$$

O11 :

$$
y \frac{\partial \mathrm{E}}{\partial z}=\mathrm{V} \mathbf{E}\left(v-\frac{k * \pi^{*}}{b^{*}}\right)
$$

Etudions d'abord le cas $y>0$. On en déduit :

$$
\frac{\sqrt{y}\|\sqrt{v}\|}{v-\frac{k-\pi^{2}}{b^{2}}}=\frac{V}{2}
$$

$\mathrm{V} 2 / \mathrm{g}$ étant positif, il faut que $y>k \pi / b$. Quand $v$ croit de $k \pi / b$ i $s, \frac{\sqrt{y^{\prime}}+\sqrt{v} H}{\nu-\frac{k^{2} \pi^{2}}{b^{2}}}$ decroit régulierement de $\infty$ à (sauf pour $k=0$ ), comme on le roil en calculant la dérivée. Pour chaque valeur de $k(\not \neq 0)$, il n'y a done qu'me racine de $x$, nous lappellerons $\alpha=$. Pour $k=0$, il se produit la singularite tronvée précédemmonl, $\nsim$ existe ou n'existe pas selon que $F \leqq 1$ (cf. $\$$ I).

D'oil, poll :

$$
\begin{gathered}
\frac{x \operatorname{lh} \alpha \mathrm{H}}{x^{2} \pi^{2}}=\frac{\mathrm{V}^{2}}{g} \\
\mathrm{~b}=\operatorname{ch} x(z+\mathrm{H}) \\
\mathrm{D}=\cos \left(\sqrt{\left.\mathrm{x}^{2}-\frac{k^{2}-\pi^{2}}{b^{2}} x+?\right)}\right.
\end{gathered}
$$

Ces expressions fournissent une premiere parlie du potentiel :

$$
W_{1} \sum_{k=0 ; 1}^{\infty} C_{k} \cos \frac{k \pi y}{b} \text { (h } x(z+H)
$$

$$
\cos \left(\sqrt{y^{2}+\frac{k-\pi^{2}}{b^{2}}} x+x\right)
$$

$v \leqslant 0$ foumil une autre partie du potentiel.

On pose :

$$
\nu=-\beta^{2}
$$

l'oì :

$$
\frac{\beta \lg \beta H}{\beta+\frac{k^{2} \pi^{2}}{b^{2}}}=-\frac{V}{g}
$$


Cette equation a bien une infinite simple et denombrable de racines pour chaque valeur de $k$. comme on s'y attendait: en effet, si $\beta$ est grand, il $y$ a une racine voisine de chaque nombre:

$$
\beta=(2 n+1) \pi / 2 \quad(n \text { entier }) .
$$

Dou la deuxieme partie de $\Phi$ :

$H_{2} \underbrace{\infty}_{k=0} \mathrm{C}_{k} \cos \frac{k \pi y}{b} \underset{\beta k}{\mathrm{E}} \mathrm{F}\left(\beta_{k}\right) \cos \beta(z+\mathrm{H})$

$$
\operatorname{ch}\left(\sqrt{\beta+\frac{k^{2} \pi^{2}}{b^{2}}} x+?\right)
$$

Evidemment :

$$
\Phi=\Phi_{1}+\Phi_{2}
$$

On démontre facilement qu'en général $\Phi_{2}$ représente une perturbation, s'eteignant comme $e-\beta a$ it partir de chaque extrémité (si le canal a une longueur $l ; \Phi_{2}$ se décompose en deux parties, l'une multipliée par $e^{-\beta x}$ et l'autre par $e^{-\beta(l-x)}$, le terme $e+\beta x$ est donc bien un terme décroissant vers l'interieur du canal).

On voit que les diverses ondes de $\Phi_{1}$ servent à "ajuster " les perturbations transversales, comme il a eté dit, alors que les perturbations de $\Phi$, servent a "ajuster " les perturbations verticales (celles-ci ont done un eflet qui "s'éleint "très vite dans le canal).

Calculons la plus petite valeur de $\beta$, qui donne la perturbation la moins amortie. On trouve $(k=0$; $\beta=0$ s'intègre dans $\Phi_{1}$ et non $\Phi_{2}$ ):

$$
\beta \geqslant \frac{\pi}{b}
$$

2. - CAS DES ONDES DE MACI QCAND HA REMARTITION DES VITESSES N'EST PAS UNIFOHME.

Nous conserverons les momes nolations que cidessus. Les equations de depart sont dans de cas:

$$
\begin{gathered}
\overrightarrow{v_{A}} \overrightarrow{\operatorname{rot}} \overrightarrow{\mathrm{V}}+\overrightarrow{\operatorname{grad}}\left(\frac{\mathrm{V}^{2}}{2}+\frac{p}{?}+g z\right)=0 \\
\operatorname{div} \overrightarrow{\mathrm{V}}=0
\end{gathered}
$$

Admettons qu'il existe dans le canal un écoulenient $\vec{V}$ voisin d'un autre écoulement $\vec{U}$ connu et donné par $\overrightarrow{\mathrm{U}}=[\mathrm{U}(z) ; 0 ; 0]$. Posons $\overrightarrow{\mathrm{V}}=\overrightarrow{\mathrm{U}}+\vec{v}$ et $\rightarrow$ considérons $\vec{v}=(u ; v ; w)$ comme une quantité très petite par rapport a $\overrightarrow{\mathrm{U}}$. J.es équations linéarisées sont alors :

$$
\begin{array}{r}
\mathrm{U} \frac{\partial u}{\partial z}+\mathrm{w} \frac{\partial \mathrm{U}}{\partial z}=\frac{1}{\rho} \frac{\partial p}{\partial x} \\
\mathrm{U} \frac{\partial v}{\partial x}=\frac{1}{\rho} \frac{\partial p}{\partial y} \\
\mathrm{U} \frac{\partial w}{\partial \boldsymbol{x}}=\frac{1}{+} \frac{\partial p}{\partial z} \\
\frac{\partial u}{\partial x}+\frac{\partial v}{\partial y}+\frac{\partial w}{\partial z}=0
\end{array}
$$

En posant $p / p=f_{x}^{\prime}$ ef en eliminant $\partial u / \partial x$, on obtient :

$$
\begin{gathered}
\mathrm{U} w=f^{\prime}+\varphi(y ; z) \\
\mathrm{U} w=f_{z}^{\prime}+\psi(y ; z) \\
-\mathbf{U} \frac{\partial v}{\partial y}--\mathbf{U} \frac{\partial w}{\partial z}+w \frac{\partial U}{\partial z}=f^{\prime \prime}
\end{gathered}
$$

P et $\psi$ étant des fonctions arbitraires d'intégration. Ces fonctions ne contenant pas $x$ peuvent être négligées si on fait abstraction des perturbations s'cteignant suivant $0 x$.

En éliminant $v$ et $f$, on trouve :

$$
\lrcorner_{3} w=\frac{\mathrm{U}^{\prime \prime}}{\mathrm{U}} w \quad \text { avec }: \mathrm{U}^{\prime \prime}=\frac{d^{\prime \prime} \mathrm{U}}{d z^{2 n}}
$$

On pent écrire :

$$
\mathrm{U}:=\mathrm{U}_{11}+\mathrm{U}_{1} z+\mathrm{U}_{22} z^{2}+\ldots
$$

$\mathrm{Si}$ on veut tenir compte de plus de trois termes, les calculs deviennent delicats; nous nous liniterous ici aux calculs faciles.

On en déduit :

$$
\Delta_{3} w=a w \quad\left(a=\frac{\mathrm{U}_{2}}{\mathrm{U}}\right)
$$

En posant :

$$
w=\mathrm{A}(x ; y) \mathrm{B}(z)
$$

el en négligeant toujours les perturbations secondaires, on obtient:

$$
\begin{gathered}
\Delta_{2} \mathrm{~A}=\lambda \mathrm{A} \\
w=\frac{\mathrm{y}}{\lambda} \operatorname{sh} \sqrt{\lambda^{2}+a}(z+\mathrm{H}) \cdot A_{\lambda} \\
I=\frac{\mathrm{V}}{\lambda}\left[\operatorname{ch} \sqrt{\lambda^{2}+a}(z+\mathrm{H})-\frac{\mathrm{U}^{\prime}}{\mathrm{U} \sqrt{\lambda^{2}+a}} \operatorname{sh} \sqrt{\lambda^{2}+a}(z+\mathrm{II})\right] \frac{A_{\lambda}}{\sqrt{\lambda^{2}+a^{2}}} \\
v=\frac{\mathrm{U}}{\lambda}\left[\operatorname{ch} \sqrt{\lambda^{2}+a}(z+H)-\frac{\mathrm{U}^{\prime}}{\mathrm{U} \sqrt{\lambda^{2}+a^{2}}} \operatorname{sh} \sqrt{\lambda^{2}+a}(z+\mathrm{H})\right] \frac{\partial A_{\lambda}}{\partial y} \frac{1}{\sqrt{\lambda^{2}+a^{2}}}
\end{gathered}
$$


On pose:

$$
\frac{\partial \mathrm{A}_{\lambda}}{\partial y}=\mathrm{B}(x) \cdot \mathrm{C}(y)
$$

D'où :

$$
\begin{aligned}
& \mathrm{C}=\sin \frac{k \pi y}{b} \\
& \mathrm{~B}=\sin \left(x \sqrt{\lambda^{2}-\frac{k^{2} \pi^{2}}{b^{2}}}+\varphi\right)
\end{aligned}
$$

La condition de surface libre est :

$$
g w=\mathrm{U}^{2} \frac{\partial u}{\partial x}=-\mathrm{U} 2\left(\frac{\partial u}{\partial y}+\frac{\partial w}{\partial z}\right)
$$

On en déduit les valeurs propres de $\lambda$ :

$$
\frac{\sqrt{\lambda{ }^{2}}+a+h \sqrt{\lambda^{2}+a}+1}{\lambda^{2}+a-\frac{k^{2} \pi^{2}}{b^{2}}}=\frac{1 \cdots}{!}
$$

Les angles de Mach sont toujours donnés par :

$$
\cos \theta=\frac{k \pi}{b \lambda}
$$

Appelons $\theta_{1}$ l'angle de Mach calculé aver a et $\theta_{2}$, langle de Мach compte tenu de la répatition non uniforme des vitesses. On en déduit :

$$
\operatorname{tg} \theta_{2}=\sqrt{\operatorname{tg}^{2} \theta_{1}-\frac{b^{2} a}{k^{2} \pi^{2}}}
$$

En pratique, $a$ est négatif, soit $a=\ldots c$, donc :

$$
\operatorname{tg} \theta_{2}=\sqrt{\operatorname{tg}^{\prime \prime} \theta_{1}+\frac{b^{2} c}{k^{2} \pi^{2}}},
$$

c'est-à-dire $0_{2.2}>0_{1}$.

Les ondes ne peuvent donc jamais être plus inclinées qu'une certaine valeur :

$$
0_{2}>\operatorname{arctg} \frac{b \sqrt{c}}{k \pi}
$$

La courbure moyenne des repartitions des vitesses est telle que $c$ est de l'ordre de :

$$
\frac{1}{3} \frac{1}{\mathrm{H}^{2}} \div \frac{1}{30} \frac{1}{\mathrm{H}^{2}}
$$

selon la rugosité des canaux (en écoulement turbulent).

\section{Cas Dr: ta nothe.}

Un calcul analogue à celui du paragraphe 1 donne :

$$
\begin{aligned}
& \Phi=\Phi_{1}+\Phi_{2}+\Phi_{3} \\
& \Phi_{1}=\sum_{k=1}^{k=k_{t}} C_{k} \cos \frac{k \pi !}{b} \operatorname{ch} \gamma(z+I) \cos \left(x \sqrt{y^{2}-\frac{k^{2} \pi^{2}}{b^{2}}}+\psi_{k}\right)
\end{aligned}
$$

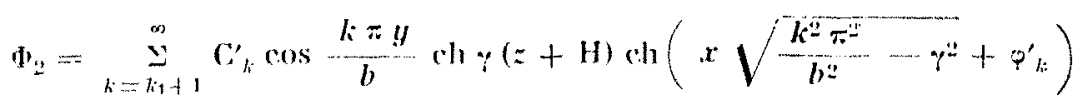

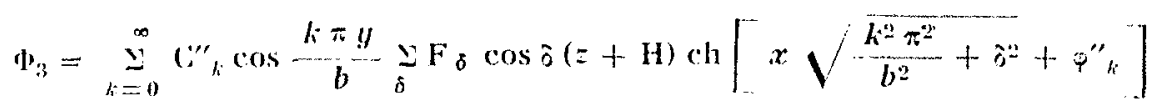

o11 :

$$
\begin{aligned}
& \gamma \operatorname{th} \gamma \mathrm{H}=\frac{\omega^{2}}{g}\left(\omega: \text { pulsation }=\frac{2 \pi}{\mathrm{T}}\right) \\
& \delta \operatorname{tg} \delta \mathrm{H}=-\frac{\omega^{2}}{g}
\end{aligned}
$$

$k_{1}$ est défini par : $\quad y^{2}-\frac{k_{1}^{2} \pi^{2}}{b^{2}} \geqslant 0 \quad$ et $\quad \gamma^{2}-\frac{\left(k_{1}+1\right)^{2} \pi^{2}}{b^{2}} \leqslant 0$

\section{IV. - CONCLUSIONS PRATIQUES DE L'ETUDE THEORIQUE}

\section{1. - Formation des perturbations du genRe} * ONDE DE MACH .

Il est clair que l'on peut injecter de l'eau dans un canal de facon à ne pas produire d'onde de
Mach : il suffit de bien choisir la distribution des vitesses à l'entréte (fig. 8).

En général, l'eau n'entre pas de cette façon dans le canal (exemple lig. 9). 
Dans la section d'entrée, la surface libre est courbe el la vitesse varie d'un point à un autre. Le ealcul montre que si certaines différences se
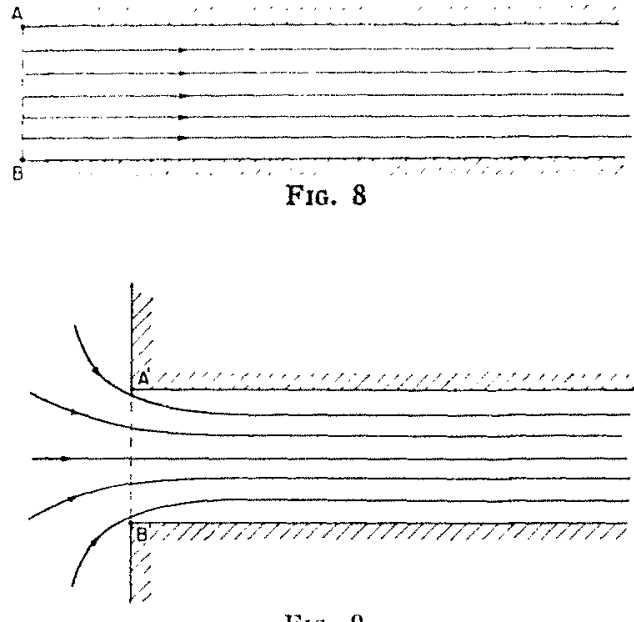

Fifi. 9

compensent, d'autres demeurent. En particulier les différences dans le sens vertical disparaissent rapidement. L'écoulement, et par conséquent la surface libre, tend vers une forme limite. Cette disparition n'est pratiquement fonction que de la largeur de l'écoulement. En pratique ce genre de perturbations ne peut pas se calculer. En effet, le calcul admet une répartition des vitesses presque uniforme, ce qui n'est justement pas réalisé dans la zone oì ces perturbations ne sont pas encore éteintes.

Les différences dans le sens horizontal donnent lieu à des perturbations qui ne s'éteignent pas (en liquide parfait), ces perturbations sont les vraies «ondes de MACH».

En fluide parfait, ces ondes dépendent en principe des deux extrémités du canal. Il faut effectivement ajuster deux constanles: amplitude et phase pour chaque onde. En pratique, quand le canal est assez long et assez rugueux, les ondes s'éteignent le long de son axe, et il se forme deux familles indépendantes de perturbations à chaque extrémité du canal, chacune ne dépendant évidemment que des conditions dans son voisinage.

Lcur cxtinction est beaucoup moins rapide que celle des ondes considérées plus haut qui s'éteignent même en fluide parfait.

\section{2. - INFLUENCE de LA RÉPARTITION DES vitesses DANS INE SFCTION.}

Dans la théric classique (shallow water theory) on ne peut pas facilement tenir compte de celle influence. Le calcul plus complet montre qu'elle intervient relativement beaucoup.
Une variation linéaire des vitesses est sans influence, par contre les dérivées supérieures, c'està-dire les détails de plus en plus poussés de la répartition, interviennent :

Exemple: Pour $\mathrm{F}=4 ; \quad b=3 y_{m} ;$ on a :

pour l'onde principale :

$$
0_{1} \# 8^{\circ}
$$

Si la rugosité est moyenne, on a, par exemple:

$$
c=\frac{1}{9 \mathrm{H}^{2}}
$$

D'où :

$$
0.22^{\circ}
$$

\section{3. - Honle.}

On voit apparaitre ici un phénomène tris curieux du point de vue mathématique (mais physiquement ivident (cf. \& 1): seules un cerhin nombre de perturbations dil genre :

$$
\cos (k \pi \eta / b) \operatorname{ch} \varphi(z+\mathrm{H}) /(x)
$$

se conservent le long du canal, les aulres s'amortissent. Les premieres comprennent la houle ordinaire $k=0$ et une houle « croisce $\$$ : la houle de Jefriers. Le nombre $k_{1}$ est le plus grand nombre qui vérifie l'inégalité :

$$
\frac{k \pi}{b}<\frac{2 \pi}{\mathrm{L}}(=\because)
$$

L chant ba longueur d'onde de la houle à crête perpendiculaire aux parois du canal ayant la pulsation (1).

Si :

$$
b<\frac{\mathrm{L}}{2}
$$

aucune houle de JeFrneys n'est possible (seul $k=0$, valeur donnant la houle ordinaire, satisfait alors à :

$$
\frac{k \pi}{b}<\frac{2 \pi}{\mathrm{L}} \text { ) }
$$

Ceci est une remarque très importante. En effet, on admet souvent en physique qu'un système est «à une dimension spatiale » si les longueurs transversales sont « petites 》 devant la longueur d'onde. Dans ce cas, "petites » signifie done plus petite que L/2 (ou plutol $L / 3$ pour que les perturbations dues aux extrémitis s'éteignent assez vite). Si on excite un systime long, par exemple un canal à houle contenant des obs- 
lacles (lig. 10), on oblient en Is soil une houle réguliere, si :

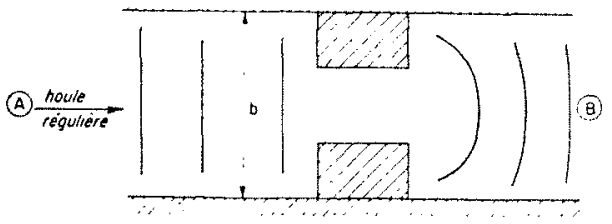

FIa. 10

$$
\left.b<\frac{\mathrm{I}}{2} \text { (figure } 2\right)
$$

soit une houle régulière plus $1 ; 2 ; \ldots k_{1}$ houles de JHFreys (figure 11) si :

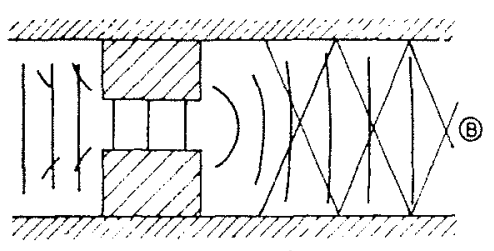

FIc. 11

$$
\left(k_{1}+1\right) \frac{\mathrm{L}}{2}>b>k_{1} \frac{\mathrm{L}}{2}
$$

soit une figure de diffraction (au sens de l'optique physique) si $k_{1}$ est tris grand (igure 12);

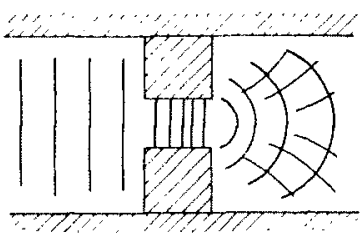

FIG. 12 soil de \& loptique gémelrique $\Rightarrow$ si hi, est immense (lig. 13).

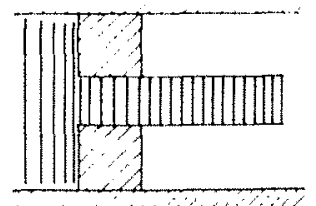

Fiti. 13

La condition $b<L / 2$ est la raison pour laquelle les guideaux des batteurs à houle doirent avoir un ecartement plus petit que $\mathrm{L} / 2$.

Si $b=(k \mathrm{~L} / 2)$, il $y$ a une résonance transversale. Elle est particulierement spectaculaire en canal convergent si la largeur du cinal passe par la valeur $k L / 2$ (en général $k=1$ ). Remarquons enfin que les houles de Jrarksys ont une célérité plus grande que les houles ordinaires.

Il y i donc tres probablement des ondes du genre « a front raide» allant plus vite que $\sqrt{ } g \mathrm{H}$ dans un canal. Expérimentalement on virifie d'ailleurs que dans un canal a houk l'eau bouge un peu, bien avant que l'onde a célérité $\sqrt{g}$ H émise par la mise en marehe du batteur ail alteint le point correspondant (en fail, cela pourrait ètre dù à d'autres phénomines que ceux étudiés ici).

On pourrait encore faire bien dautres remarques à ce sujet, en partieulier évaluer les parasites dus a un balleur dont les sections horizontales ne sont pas parfailement des droites perpendiculaires a laxe du canal; ou fludier les hatleurs « serpents».

\section{R. MsYin,}

Inginieur an Laboraloire Datuphinots d'Hydratique (Neypic-(irenoble) (")

Avant de publier ces longs commentaires de M. MیYsn, nous en amions fait part aux auleurs de

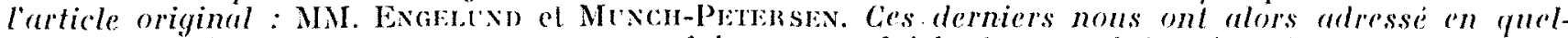
ques lignes des remarques que nous nous faisons un plaisir de reproduire ci-apres.

It has been a pleasure fur us to read the many interesting comments given by Mr. Mryis in a letter of November 3 , and we should like to add the following remarks.

In Aerodynamics it seems common to make a distinction between "Mach-waves" and "shockwaves". The Mach-waves may in principle be accounted for by the lincarized equations, hecause the loss of head is negligible, while the computation of the shock-waves is generally las more complicated as they are characterized by a considerable increase of entrophy.
C'est avec plaisir que nous avons pris connaissance de tous les interessints commentaires contenus dans la leftre de M. Mhren du 3 novembre dernier, of nous aimerions $y$ ajouler les; quelques remarques suivantes.

En acrodynamique, on lail couramment la distinction entre les «ondes de Mach $»$ et les « ondes de choc». Les "ondes de Mach» pewrent, en principe, être lraitées par des équations linéarisées, car la perte de charge est négligeable; par contre, les calculs concernant les eondes do choc "sont generalement beatucoup plus compli- 
In Hydraulics the conditions are quite sinilas. It would cerlainly be correct to make a distinclion between waves of small amplitude and raal "shock-waves" which are nothing but oblique hydraulic jumps. However, such a distinction does not seem to be conventional. The transition between the two wave-forms is quite gradual.

Considering the fact that the theory of steady now in an infinitely long channel with infinitesimal perturbations (the theory developed in our paper) is in good agreement with the experiments, we must conclude that in these experiments the waves are not real "shockwaves", since they can be accounted for without considering the loss of head.

This is remarkable because the wave fronts are relatively sharp, and the amplitudes relatively great (compare fig. 8), so that it would be natural in the first instance to talk about "shock fronts".

Perhaps the insufficiency of the traditional shock-wave considerations are most strikingly demonstrated by our fig. 9 . It is usually suj posed that the waves are crossing the channel heing reflected from the walls.

In fig. 9 and 10 it will be seen that a wave may disappear at the wall, a new wave front being formed a little downstream at the opposite wall. ques, car elles sont caractérisces par une considirable augmentation de $l$ " entropic .

En hydraulique, il en va lout à fait de même. Il serait certainement correct de distinguer les ondes de petite amplitude et les véritables « ondes de choc » qui ne sont autres que des ressauts obliques. Cependant, une telle distinction ne parait pas courammenl admise. La transition entre ces denx types d'ondes est tris graduelle.

Comple tenu de ce que la théorie de l'écoulement permanent dans un canal infiniment long, siege de perturbations trés petites (c'est la théorie que nous développons dans notre article) est en bon accord avec les résultats expérimentaux, nous devons conclure que, dans ces essais, les ondes ne sont pas de véritables " ondes de choc », puisqu'elles peuvent ìtre traitécs sans tenir compte de la perte de charge.

Ceci est remarquable, car les fronts d'ondes sont relativement raides, et les amplitudes relatirement grandes (notre figure 8 ), si bien qu'il serait normal, dans le premier exemple, de parler de « fronts de choc».

Pent-être l'insuffisance des dóveloppements classiques sur les « ondes de choc » apparaissent-il plus clairement dans notre figure 9. On suppose, généralement, que les ondes traversent le canal après réflection sur les parois.

Sur les figures 9 et 10 , on verra qu'une onde peut s'éteindre contre la paroi, un nouveau front l'ondes prenant naissance sur la paroi opposée, un peu à l'aval.

Frank ENGELrivis.

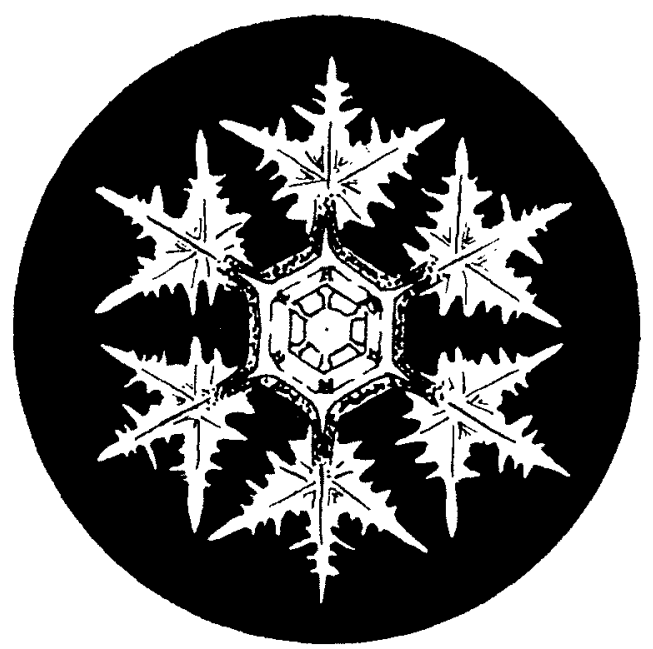

